erythromycin, ciprofloxacin, penicillin and tetracycline respectively. Resistance for ciprofloxacin had rise from $56 \%$ in $2012,58.8 \%$ in $2013,66.7 \%$ in 2014 to $68.5 \%$ in 2015 .

Conclusion Spectinomycin, cefixime, ceftriaxone, azithromycin are useful. Ciprofloxacin the most prescribed antibiotic is no longer reliable for treatment of GC. Continuous surveillance is essetial to mordify treatmet guidelies. Worsening GC drug resistance will compromise effective treatment and decrease disease control efforts.

\section{P2.31 ANNULAR LICHEN PLANUS ON PENIS TREATED WITH TOPICAL PIMECROLIMUS $1 \%$}

${ }^{1}$ Natasatsekova, ${ }^{2}$ Krasnaliev, ${ }^{2}$ I Bakardjiev, ${ }^{1}$ L Miteva, ${ }^{1} \mathrm{G}$ Pehlivanov. ${ }^{1}$ Medical University, Department of Dermatovenereology, Sofia - Bulgaria; ${ }^{2}$ Medical University, Department of Dermatovenereology, Varna - Bulgaria

\subsection{6/sextrans-2017-053264.207}

Introduction Lichen planus (LP) is an idiopathic inflammatory disease of the skin and mucous membranes. Classical LP is characterised by pruritic, violaceous papules that favour the extremities. Annular lichen planus (ALP) is a long-recognised clinical variant of lichen planus, but is often considered uncommon in occurrence. ALP commonly involves the male genitalia but also has a predilection for intertriginous areas such as the axilla and groin folds. Distal aspects of the extremities, and less commonly the trunk, may also be involved.

Methods We report the case of 38 year-old uncircumcised male patient who addressed our clinic for multiple asymptomatic annular lesions on the glans penis and corpus penis and whitish linear bilateral and symmetric lines on buccal mucosa with 2 years duration. During this period the patient was treated several times with antifungal and corticosteroid drugs without improvement.

Results Diagnosis was based on clinical features and histological examination. The patient was treated with topical pimecrolimus $1 \%$ with significant improvement after 3 weeks.

Conclusion This description highlights the importance of patients presenting annular lesion on penis be routinely required to undergo further medical examination for Canddida spec., Sexually transmitted infections and if is necessary to perform the biopsy because the exact diagnosis is basis for proper treatment.

\section{P2.32 SYPHILIS MANAGEMENT IN CHILE: IS PARTNER NOTIFICATION A MISSED OPPORTUNITY?}

${ }^{1}$ Nicole Iturrieta, ${ }^{1}$ Meredith Temple-Smith, ${ }^{2}$ Jane Tomnay. ${ }^{1}$ Department of General Practice University of Melbourne, Melbourne, Australia; ${ }^{2}$ Department of Rural Health - Centre for Excellence in Rural Sexual Health - University of Melbourne, Melbourne, Australia

\subsection{6/sextrans-2017-053264.208}

Introduction Partner Notification (PN) is an essential strategy for sexually transmitted infection (STI) control, but both policies and methods of implementation vary among countries. Using syphilis as a case study, we investigated the Chilean policy regulations relating to $\mathrm{PN}$ and examined the effect of these policies in the field.

Methods Qualitative techniques were used to gain insights into current Chilean policies related to syphilis management and control, and to understand the nuances of delivering $\mathrm{PN}$ in the current system. The latest national standards/policies with the words "STIs" or "syphilis" in the title were analysed. 48 semi-structured face-to-face interviews were conducted with healthcare providers (HCP). A third of the interviews were transcribed verbatim and translated from Spanish to English for thematic analysis, which followed an inductive approach based on grounded theory. Following the identification of themes, remaining interviews were coded utilising a method of constant comparison to highlight concordance and dissonance of participant views.

Results A total of six documents met the inclusion criteria. While syphilis prevention is highlighted in them, PN was barely acknowledged as a necessary activity to reduce the risk of transmission and reinfection. No document provided detailed information about PN strategies. HCP recognised PN as an essential strategy for STI control; however, they identified a lack of available guidelines and resources to ensure best practice. Additionally, the PN strategies currently undertaken are inconsistent and varied across services.

Conclusion Strengthening policies at a local and national level to reinforce PN should be considered by Chilean authorities. A priority action plan which includes training of HCP in PN and a strong support network for efficient delivery of PN would both enhance STI control and the long-term impact of existing policies.

\section{P2.33 CO-OCCURRENCE OF TRICHOMONAS VAGINALIS AND BACTERIAL VAGINOSIS AMONG WOMEN; PREVALENCE AND TREATMENT OUTCOMES}

${ }^{1}$ Atricia Kissinger, ${ }^{2}$ Christina Muzny, ${ }^{3}$ Leandro Mena, ${ }^{4}$ Rebecca Lillis, ${ }^{2}$ Jane R Schwebke, ${ }^{5}$ Laura Beauchamps, ${ }^{4}$ Stephanie N Taylor, ${ }^{1}$ Norine Schmidt, 'Lauren Ostrenga, ${ }^{6}$ David H Martin. 'Tulane University School of Public Health and Tropical Medicine - Department of Epidemiology, New Orleans, USA; ${ }^{2}$ University of Alabama at Birmingham, Division of Infectious Diseases, Birmingham, USA; ${ }^{3}$ Univeristy of Mississippi Medical Centre Department of Medicine, Jackson, USA; ${ }^{4}$ Louisiana State University Health Sciences Centre Section of Infectious Diseases, New Orleans, USA; ${ }^{5}$ University of Mississippi Medical Centre - Department of Medicine, Jackson, USA; ${ }^{6}$ Tulane University School of Public Health and Tropical Medicine - Department of Epidemiology, New Orleans, USA

\subsection{6/sextrans-2017-053264.209}

Introduction Both Trichomonas vaginalis (TV) and bacterial vaginosis $(\mathrm{BV})$ cause vaginitis and place women at higher risk for HIV infection. Both are treated with metronidazole (Mtz) but at different doses. The purpose of this study was to examine the co-occurrence of these infections and BV treatment outcomes among $\mathrm{TV}+/ \mathrm{BV}+$ women multi-dose $\mathrm{Mtz}$ for the treatment of TV.

Methods Women attending three sexually transmitted disease clinics in the southern US who had a diagnosis of TV (culture or NAAT confirmed) were interviewed and examined for BV using a Nugent score $\geq 7$. Women were randomised to either $2 \mathrm{~g}$ single dose or $500 \mathrm{mg} \mathrm{Mtz}$ BID for 7 days multi-dose for the treatment of TV and followed 3-12 weeks post TV treatment and retested for both TV and BV. Medical records were abstracted for Amsel criteria for a subset of the cohort.

Results Of $528 \mathrm{TV}+$ women at baseline, $49.8 \%$ also had BV per Nugent score, $44.3 \%$ reported a history of BV and $5.9 \%$ also had yeast. Of 289 women whose medical records were abstracted, 23.5\% had a vaginal discharge consistent with BV (i.e. thin and white/grey), and $34.1 \%$ were BV+ per Amsel at baseline. Of the 46 women who were $\mathrm{BV}+$ at baseline per Amsel (i.e. diagnosed at point of care) and per Nugent (i.e. 